\title{
Recurrent abdominal pain in school children in a rural tertiary hospital
}

\author{
Prabhu S.G. ${ }^{1}$, Abraham G. ${ }^{2}$, Nair L.M. ${ }^{3}$ \\ ${ }^{1}$ Dr. Satish G. Prabhu, Professor Consultant Paediatric Surgeon and unit Chief, ${ }^{2}$ Dr. George Abraham, Assistant \\ Professor, ${ }^{3}$ Dr. Lakshmi Malavika Nair. All authors are affiliated with the Department of General Surgery, MOSC \\ Medical College, Kolenchery, Medical College Road, PO Kolencherry, Kochi, Kerala, India.
}

Corresponding Author: Dr. George Abraham, Assistant Professor, Department of General Surgery, MOSC Medical College, Kolenchery, Medical College Road, PO Kolencherry, Kochi, Kerala, India. Email: drgeorgeabr@gmail.com

\begin{abstract}
Background: Recurrent abdominal pain is one of the most common presenting complaints among school going children, with a prevalence of $10-12 \%$. Most of the children with recurrent abdominal pain are not evaluated further, either due to the mild abdominal pain or transient nature of the symptoms. Recurrent abdominal pain requires complete evaluation to look for organic causes. However, in many cases, where no specific organic cause can be identified, a psychosomatic component has been shown to play a role. The aim of the study is to evaluate children with recurrent abdominal pain in the age group 2-12 years for a cause at a tertiary rural hospital, in an attempt to better understand this syndrome. Methods: Retrospectively collected data from 210 consecutive patients aged between 2-12 years from January 2015 to January 2017, who presented to Department of Surgery and Emergency department in MOSC Medical College with recurrent abdominal pain were studied. Data collection included demographic details, symptomatology and clinical details, and response to treatment. Each patient was thoroughly evaluated to identify an organic cause for the pain. All the patients were evaluated by the paediatrician for any underlying medical cause. Results: During the two-year period, 210 patients were evaluated and investigated, and an organic cause was detected in 44 patients, while 166 patients were diagnosed to have RAP. The 44 patients with an organic cause were treated for the primary condition and were asymptomatic on follow up. Investigations revealed that 20 patients suffered from acute appendicitis, 20 patients were diagnosed with habitual constipation Patients without an organic cause were reassured, counselled and treated symptomatically. All the patients were on follow up for the entire period of the study. Of the 166 patients diagnosed with RAP, 110 patients responded to symptomatic measures, while 21 patients were referred to child psychiatry and are on long term follow up. Conclusion: Recurrent abdominal pain is a significant cause of functional impairment in the life of a child. Thorough evaluation of the patient to rule out organic causes is vital. Majority of the children diagnosed with recurrent abdominal pain respond to counselling. Acknowledging the existence of the symptoms instead of dismissing it, and empathising with the child is often all that is required in the management of these patients
\end{abstract}

Keywords: Recurrent abdominal pain, Childhood abdominal pain, Non organic pain abdomen

\section{Introduction}

One of the most common presenting complaints among school going children is recurrent abdominal pain (RAP). Despite a prevalence of 10-12\% among school going children [1] nearly half of all children with recurrent abdominal pain do not seek medical care for it [2]. This can be attributed to the mild severity or transient nature of the symptoms. Medical aid is sought for only when the pain impairs the daily functioning of the child or family [3]. RAP is defined as a syndrome

Manuscript Received: $4^{\text {th }}$ September 2019

Reviewed: $14^{\text {th }}$ September 2019

Author Corrected: $20^{\text {th }}$ September 2019

Accepted for Publication: $24^{\text {th }}$ September 2019 presenting with at least three episodes of abdominal pain, severe enough to affect the activities of the affected children over a period longer than three months [1]. Often presenting as acute abdominal disorder with periumbilical pain which is associated with autonomic and functional symptoms like nausea, vomiting, pallor and headache [4], RAP warrants prompt and extensive evaluation to diagnose the cause of the symptoms. An organic cause for RAP is difficult to diagnose, however, in some studies, the percentage of organic RAP was found to be as high as $82 \%$ [5]. On the other hand, a recent epidemiological study in Sri Lanka diagnosed an 


\section{Original Research Article}

organic cause for RAP only in $23.6 \%$ of affected children $[5,6]$. The study is important because in a vast majority of cases, with multiple episodes of recurrent abdominal pain a treatable clear organic cause could not be diagnosed and some cases where surgical procedures like appendectomy was done on clinical grounds the abdominal pain still persisted. This study highlights the clinical relevance of recurrent abdominal painnonorganic, and the role of cause's psychosocial component as described a number of studies.

\section{Material and Methods}

Study Setting: The study was conducted in department of General Surgery MOSC Medical College, Kolenchery

Aim: Evaluation of children with recurrent abdominal pain in the age group 2-12 years for a cause of abdominal pain at a tertiary rural hospital

Study Design: Retrospective observational study

Study Period: Between January 2015 and January 2017

Inclusion Criteria: 210 Cases Recurrent abdominal pain patients presenting Patients with abdominal pain of more than 3 months who presented to casualty and surgical OP were included in the study
Exclusion: Children with other morbidities like epilepsy, febrile conditions, previously diagnosed congenital conditions like genitourinary anomalies and GI anomalies and those who were not willing for the study were excluded

Ethical Consideration: The study was approved by the institutional human ethics committee.

Study Design: Retrospectively collected data from 210 consecutive patients with recurrent abdominal pain aged between 2 and 12 years were studied. Each patient was thoroughly evaluated by clinical examination, blood investigations and imaging, and response to treatment, to identify an organic cause for the pain. All the patients were evaluated by the paediatrician for any underlying medical cause. Patients in whom an organic cause could not be identified after thorough evaluation were treated with symptomatic measures and reassurance, and refractory cases were referred to department child psychiatry. All children were kept on close follow up to monitor their response to treatment

Statistical analysis: This study was primarily a qualitative analysis. Data was analysed using Microsoft Excel and the prevalence was estimated as percentages. Calculation of statistical significance will be carried out by chi- square test for univariate analysis and bivariate analysis.

\section{Results}

\begin{tabular}{|c|c|c|c|}
\hline Age & Males & Females & Total \\
\hline $2-6$ years & 90 & 20 & 110 \\
\hline $6-12$ years & 60 & 40 & 100 \\
\hline \multicolumn{4}{|l|}{ Associated symptoms } \\
\hline Nausea & 190 & & \\
\hline Vomiting & 30 & & \\
\hline Bloating & 80 & & \\
\hline Diarrhea & 43 & & \\
\hline Constipation & 100 & & \\
\hline Anorexia & 35 & & \\
\hline \multicolumn{4}{|l|}{ Blood investigations } \\
\hline Anemia & 20 & & \\
\hline Lymphocytosis & 40 & & \\
\hline Raised ESR & 20 & & \\
\hline Deranged LFT & 5 & & \\
\hline \multicolumn{4}{|l|}{ Diagnosis } \\
\hline Recurrent appendicitis $(\mathrm{n}=20)$ & Male & Female & \\
\hline $2-6$ years & 7 & 3 & \\
\hline $6-12$ years & 8 & 2 & \\
\hline Habitual constipation & 15 & 5 & \\
\hline Malrotation without volvulus & 1 & & \\
\hline Suspected extraluminal obstruction & 3 & & \\
\hline
\end{tabular}




\section{Original Research Article}

Patients with frequent episodes of abdominal pain not less than 3 episodes over a period of 3 months were followed up in $\mathrm{OP}$, and patients with frequent episodes of abdominal pain were admitted and investigated. Investigations revealed that 20 patients suffered from acute appendicitis, of which 15 were male ( 7 patients were between 2-6years of age, 8 patients were aged between 7-12 years), and 5 were female ( 3 patients were between 2-6years of age, 2 patients were aged between $7-12$ years)

60 patients presented with a clinical history of acute abdomen, of which 40 were male, and 20 were female. 190 patients had nausea, however, only 30 patients complained of vomiting. 80 patients complained of bloating, and 43 patients felt anorexic. A 100 patients complained of constipation, while 25 patients suffered from diarrhoea.

Detailed investigation revealed 20 patients were anaemic. Differential counts showed leucocytosis in 60 patients, and lymphocytosis in 40 patients. An elevated ESR was seen in 20 patients. Elevated LFT was seen in 5 patients. However, none of the patients had abnormal RFT or URE reports. Ultrasound abdomen revealed mesenteric lymphadenopathy in 110 patients.

Barium meal and follow through revealed malrotation without volvulus in 1 patient, a 4-year-old boy, and 3 patients were suspected to have extraluminal obstruction.

Diagnostic laparoscopy was done in these 3 patients, which revealed no bands or extraluminal obstruction. 20 patients were diagnosed with habitual constipation, of which 15 were male (12 patients were between 2-6years of age, 3 patients were aged between 7-12 years), and 5 were female (3 patients were between 2-6years of age, 2 patients were aged between 7-12 years). All the patients were evaluated by the paediatrician for any underlying medical cause. Patients without an organic cause were reassured and counselled and treated symptomatically. All the patients were on follow up for the entire period of the study, and an organic cause was detected in 44 patients, while 176 patients were treated symptomatically. The 44 patients with an organic cause were treated for the primary condition and were asymptomatic on follow up. Of the 155 patients diagnosed with RAP, 110 patients improved with symptomatic treatment and reassurance, while 21 patients were referred to child psychiatry and are on long term follow up.

\section{Discussion}

Recurrent abdominal pain is a common presenting complaint in school going children. Defined by Apley as a syndrome presenting as a minimum of three episodes of abdominal pain occurring in the space of three months, severe enough to affect daily activities, RAP forms a significant proportion of patients presenting to the paediatric $\mathrm{OP}$.

Although RAP is reported in $10-12 \%$ of school going children in developed countries [7], nearly half of them do not seek medical care. Among those that do seek medical attention, diagnosing an organic cause for the pain remains to be a challenge, with the percentage of detectable cause of abdominal pain ranging anywhere from $23 \%$ [7] to as high as $82 \%$ [5]. In our case $44 / 210$ $(20.95 \%)$ cases showed organic cause of recurrent abdominal pain.

Recurrent abdominal pain can further be classified by symptomatology, according to Rome II criteria, as functional dyspepsia, irritable bowel syndrome, functional abdominal pain, abdominal migraine and aerophagia. Organic causes for abdominal pain are numerous, including urinary tract infection, inflammatory bowel disease and obstructive uropathy to name a few [8]. Furthermore, epidemiological studies based on different demographics have shown the most common cause for RAP in India is Giardiasis [9], while in Sri Lanka, it is constipation [6]. However, we did not find any organic cause other than constipation as a cause for recurrent abdominal pain.

Meanwhile, in many developed countries, the common organic causes include chronic constipation and gastroesophageal reflux disease. [10]. Those without organic causes were diagnosed to have functional gastrointestinal disorder. The purpose of classifying non-organic RAP into the appropriate functional bowel disorder is to help the child and the parents know that the symptoms they are feeling are real but not dangerous or life threatening.

Often a simple explanation of the condition and reassurance is enough to relieve the anxiety of the child and the family once the diagnosis is made. Recurrent abdominal pain is so common, nearly all school children experience it. While most cases have no identifiable organic cause, many theories have been postulated to explain the root cause of RAP. One such postulate is called "visceral hyperalgesia", described by 


\section{Original Research Article}

Di Lorenza et al [11] as an alteration in the perception of visceral sensation due to changes in the 'brain-gut axis' brought about by psychological stress. Another concept is that of abdominal migraine, a concept supported by positive response to anti-migraine treatment in certain cases. [12]. In a study conducted by Hotopf, children with recurrent abdominal pain were found to have anxiety disorders as adults, more frequently as compared to the general population [13].

In the present study, more than half of the patients improved after symptomatic measures and reassurance (110/210) 52.3\%. Reassurance with an emphasis on rehabilitation remains the mainstay of treatment. This includes acknowledging the fact that the pain is indeed real, and to attend to it with the same level of concern.

Simultaneous recognition and management of coexisting potentially contributory factors like habitual constipation and stress forms a part of the wholesome treatment of RAP. Refractory cases require psychological intervention in the form of cognitivebehavioural therapy to modify thoughts, beliefs and behavioural responses to symptoms and effects of illness [14]. Other treatment modalities include biofeedback, relaxation therapy, coping skills training, hypnosis and family therapy. Long term follow up may be required in most of these patients and can be reassuring to the patient and the caretakers. The main limitation of this study was unavailability of tests required to diagnose more common diseases in western countries like coeliac disease, Practical difficulty of conducting endoscopy for diagnosing certain conditions like $\mathrm{H}$ pylori gastritis, eosinophilic gastritis and medical conditions like porphyria.

\section{Conclusion}

Recurrent abdominal pain is a significant cause of functional impairment in the life of a child, and is a source of stress for the parents. The inability to define a cause and the persistence of symptoms despite multiple attempts at treatment make it an unappealing diagnosis in the eyes of the caretaker.

However, thorough evaluation of the patient ruling out organic causes often provides some relief to the parent, and a majority of the children diagnosed with recurrent abdominal pain respond to symptomatic measures, reassurance and counselling.

Acknowledging the existence of the symptoms instead of dismissing it and empathising with the child is often all that is required in the management of these patients.

\section{What the study adds to the existing knowledge?}

Even though non organic causes is attributed to Recurrent abdominal pain in children, with more frequent uses of imageology and judicious use of lab investigation quite often organic cause are detected in RAP. In our case $20 \%$ were detected to have organic cause in contrast to the reported cases of $10-12 \%$.

\section{Author's contribution}

Dr. Satish G Prabhu: Principal investigator

Dr. George Abraham: Co-investigator, manuscript preparation

Dr. Lakshmi Malavika Nair: Patient coordination

Acknowledgement- Authors would like to thank the Department of Paediatrics, M.O.S.C Medical College

\section{Declaration}

Conflict of interest: None declared.

Funding: Nil, Permission from IRB: Yes

\section{References}

1. Apley J, Naish N. Recurrent abdominal pains: a field survey of 1,000 school children. Arch Dis Child. 1958;33(168): 165-170. doi: 10. 1136/adc. 33. 168. 165

2. Boey CC, Goh KL. Recurrent abdominal pain and consulting behaviour among children in a rural community in Malaysia. Dig Liver Dis.2001; 33(2):140144. doi: https ://doi. org/10. 1016/ S1590-8658 (01) 80069-4.

3. Plunkett A, Beattie RM. Recurrent abdominal pain in childhood. J R Soc Med. 2005;98(3):101-106. doi: 10. 1258/jrsm.98.3.101

4. Nagal L, Dabi J, Mourya HK. A clinicoepidemiological study of functional pain abdomen in children. Int J Med Biomed Stu. 2019;3(9):1-3. doi: 10. 32553/ijmbs.v3i9.512

5. Buch NA, Ahmad SM, Ahmed SZ, Ali SW, Charoo BA, Hassan MU. Recurrent abdominal pain in children. Indian Pediatr. 2002;39(9):830-834.

6. Devanarayana NM, de Silva DG, de Silva HJ. Aetiology of recurrent abdominal pain in a cohort of Sri Lankan children. J Paediatr Child Health. 2008;44(4): 195-200. doi: 10.1111/j.1440-1754.2008.01295.x. 


\section{Original Research Article}

7. Huang RC, Palmer LJ, Forbes DA. Prevalence and pattern of childhood abdominal pain in an Australian general practice. J Paediat Child Health. 2000;36(4): 349-353. doi: https://doi.org/10.1046/j.1440-1754.2000. 00513.x.

8. Pearl RH, Irish MS, Caty MG, Glick PL. The approach to common abdominal diagnoses in infants and children: part II. Pediatr Clinic. 1998;45(6): $1287-$ 1326.doi: https:/ /doi.org/10.1016/ S0031-3955(05) 700 92-4

9. Balani B, Patwari AK, Bajaj P, Diwan N, Anand VK. Recurrent abdominal pain - a reappraisal. Indian Pediatr. 2000;37(8):876-881.

10. Størdal K, Nygaard EA, Bentsen B. Organic abnormalities in recurrent abdominal pain in children. Acta Paediatr. 2001 ;90(6): 638-642. doi: https://doi. org/10.1111/j.1651-2227.2001.tb02426.x.
11. Di Lorenzo C, Youssef NN, Sigurdsson L, Scharff L, Griffiths J, Wald A. Visceral hyperalgesia in children with functional abdominal pain. J Pediatr. 2001;139 (6): 838-843. doi: https://doi.org/10.1067/mpd.2001.118883

12. Symon DN, Russell G. Double blind placebocontrolled trial of pizotifen syrup in the treatment of abdominal migraine. Arch Dis Child. 1995;72(1):48-50. doi: 10.1136/adc.72.1.48

13. Hotopf M, Carr S, Mayou R, Wadsworth M, Wessely S. Why do children have chronic abdominal pain, and what happens to them when they grow up? Population based cohort study. BMJ. 1998;316(7139): 1196-200. doi: 10.1136/bmj.316.7139.1196.

14. Janicke DM, Finnev JW. Empirically supported treatments in pediatric psychology: recurrent abdominal pain. J Pediatr Psychol. 1999; 24(2):115-127. doi: 10. 1093 / jpepsy/24.2.115.

\section{How to cite this article?}

Prabhu S.G, Abraham G, Nair L.M. Recurrent abdominal pain in school children in a rural tertiary hospital. Surgical Update: Int J surg Orthopedics. 2019;5(4):266-270.doi:10.17511/ijoso.2019.i04.06 